\title{
How Varying the Dipole Lengths of a Uniform Linear Array Affects the Performance of an ESPRIT-based Direction Finding Algorithm
}

\author{
GERALD PACABA ARADA \\ Electronics and Communications Engineering Department \\ De La Salle University \\ 2401 Taft Avenue, Malate, 1004 Manila \\ PHILIPPINES \\ Received: October 15, 2020. Revised: November 27, 2020. Accepted: November 30, 2020. \\ Published: December 11, 2020.
}

\begin{abstract}
Uniform linear array of identical dipoles is popularly used to collect data for the direction-of-arrival estimation of an incident source. Since the dipole elements of the uniform linear array are electromagnetically and mutually coupled, the presence of mutual coupling may degrade the performance of any direction finding algorithms. In order to mitigate mutual coupling, several references introduced modifications of the well-known ESPRIT algorithm. These modifications involve discarding the data collected by linear array at the two ends. However, the assumption of several literature that the mutual coupling matrix to be both Toeplitz and banded are invalid. This is pointed out by the "method of moments" based computer simulation tool used in this paper. The Toeplitz-and-banded coupling-matrix assumption leads to the mutual coupling being mis-modeled. Furthermore, previous studies failed to consider how varying the dipoles' electrical length affect the performance of the direction-of-arrival estimation under mutual coupling.
\end{abstract}

Keywords: uniform linear array, antenna array mutual coupling, array signal processing, direction-of-arrival estimation, electromagnetic coupling, mutual coupling.

\section{INTRODUCTION}

ESPRIT (Estimation of Signal Parameters via Rotational Invariance Technique)[1] is an algorithm that is based on subspace method. This eigenstructurebased technique has gained much attention and has been proven to be an effective approach in directionof-arrival (DOA) estimation. The straightforward implementation of ESPRIT can be attributed to the sensor array's inherent translational invariance, taking advantage of the rotational invariance of the signal subspaces. However, the presence of mutual coupling in an antenna array leads to the degradation of the direction finding performance of ESPRIT [2, 3, 4].
Previous studies introduced modifications to ESPRIT, in order to mitigate the effects of mutual coupling. In [5, 6, 7, 8, 9, 10, 11], the mutual coupling from the empirical data are compensated before applying the ESPRIT algorithm and requires prior knowledge of the exact mutual coupling matrix.

Similar to the algorithm adopted in this paper, $[2,12,15,116,177,18,19,20,21]$, discard the antennas from either end of the uniform linear array. Data from the overlooked antennas are not fed to ESPRIT. Only data from the trimmed array or the middle antennas are further processed and would have an array manifold that is suited for ESPRIT due to their rotational invariance mathematical structure. However, such method presumes that the mutual coupling matrix follow a Toeplitz and banded algebraic structure.

Uniform linear array is mainly used for beamforming and direction-of-arrival estimation. The array is composed of identical and equally spaced dipoles positioned along a straight line with uniform feed system. Electrically short (i.e. $L<\underset{10}{\lambda}$ ) and long dipole antennas can be used for direction finding. Electrically long dipoles have been investigated for direction finding in [22, 23, 24] but those dipoles' perpendicularity precludes the mutual coupling effects investigated in this paper.

This paper will not only address the issue on the Toeplitz and banded properties of the mutual coupling matrix, but will also investigate the effects of varying the electrical length of the dipoles with identical properties and orientation in a uniform linear array.

\section{II.IULA'S DATA MODEL FOR A UNIFORM LINEAR ARRAY OF SENSORS THAT ARE NOT ELECTROMAGNETICALLY COUPLED}

Consider $K$ number of identical isotropic sensors, lying on the $x$-axis, with a uniform spacing of $\Delta$, an identical orientation, and no electromagnetic coupling among them. Suppose a narrowband plane-wave $s(t)$, of wavelength $\lambda$ and power $\mathcal{P}$, impinges upon the aforementioned array at a direction-of-arrival (DOA) of $\theta \in[0, \pi)$ defined with respect to the array axis. 
This $s(t)$ would register a complex-phase shift of $e^{j 2 \pi \frac{x_{\ell}}{\lambda} u}$ at the $k$ th sensor, relative to an hypothetical sensor at the $x$-coordinate's origin. In the above, $u:=\sin (\theta)$ denotes the Cartesian direction-cosine along the $x$-axis,

The entire array's observed data, at the time $t$, may be represented as a $K \times 1$ vector,

$$
\overbrace{\left[\begin{array}{c}
z_{1}(t) \\
\vdots \\
z_{K}(t)
\end{array}\right]}^{\mathbf{z}(t):=}=\overbrace{\left[\begin{array}{c}
e^{j 2 \pi \frac{\Delta}{\lambda} u} \\
\vdots \\
e^{j 2 \pi \frac{\Delta}{\lambda} K u}
\end{array}\right]}^{\mathbf{a}(\theta):=} s(t)+\overbrace{\left[\begin{array}{c}
n_{1}(t) \\
\vdots \\
n_{K}(t)
\end{array}\right]}^{\mathbf{n}(t):=},
$$

where $n_{k}(t)$ refers to the complex-valued zero-mean additive white noise at the $k$-th sensor at time $t$ and possesses power $\mathcal{P}_{n}$. The above data model has only one incident signal, so that the subsequent development may focus on the impact of inter-antenna electromagnetic coupling on ESPRIT-based direction finding.

With $N$ snapshots taken at the distinct instants of $\left\{t_{n}, n=1, \ldots, N\right\}$, the entire data set may be represented as a $K \times N$ matrix,

$$
\mathbf{Z}:=\left[\mathbf{z}\left(t_{1}\right), \ldots, \mathbf{z}\left(t_{N}\right)\right] .
$$

The direction finding (DF) problem aims to estimate all $\theta$ from $\mathbf{Z}$.

\section{REVIEW OF ( 635,7}

The ESPRIT algorithm [I] has its key algorithmic steps summarized below for the degenerate case of a single-source scenario.

(i) From the dataset $\mathbf{Z}$ in (2), form the $K \times K$ data spatial correlation matrix $\mathbf{Z Z} \mathbf{Z}^{H}$, where the superscript ${ }^{H}$ symbolizes the Hermitian operation.

(ii) Compute the principal eigenvector $\mathbf{e}$ of $\mathbf{Z Z}^{H}$. This $K \times 1$ principal eigenvector spans the rank1 signal subspace of the data spatial correlation matrix.

(iii) Delete the top element of the $K \times 1$ vector $\mathbf{e}$ to form an $(K-1) \times 1$ vector, $\mathbf{e}_{1}$.

(iv) Delete the bottom element of the $K \times 1$ vector $\mathbf{e}$ to form an $(K-1) \times 1$ vector, $\mathbf{e}_{2}$.

(v) Compute $\psi:=\mathbf{e}_{1}^{H} \mathbf{e}_{2}$.

(vi) Compute $\hat{u}:=\frac{/ \phi}{2 \pi \Delta / \lambda}$ as the estimate of the incident emitter's Cartesian direction-cosine $u$.

(vii) Compute $\hat{\theta}:=\sin ^{-1} u$ as the estimate of the incident emitter's direction-of-arrival $\theta$.
Step (iii) above corresponds to ignoring the leading sensor's data, whereas step (iv) above corresponds to ignoring the trailing sensor's data. That is, these two steps form two $(K-1)$-sensor subarrays out of the overall $K$-sensor array. The former and the latter would, respectively, have the $(K-1) \times 1$ subarray manifolds,

$$
\begin{aligned}
& \mathbf{a}_{1}(u)=\left[e^{j 2 \pi \frac{\Delta}{\lambda} u}, \cdots, e^{j 2 \pi \frac{\Delta}{\lambda}(K-1) u}\right]^{T}, \\
& \mathbf{a}_{2}(u)=\left[e^{j 2 \pi \frac{\Delta}{\lambda} 2 u}, \cdots, e^{j 2 \pi \frac{\Delta}{\lambda} K u}\right]^{T},
\end{aligned}
$$

where the superscript ${ }^{T}$ denotes a transposition. These two subarrays' manifolds are mathematically identical except for a "rotational invariance" of $e^{j 2 \pi \frac{\Delta}{\lambda} u}$, which embeds the to-be-estimated parameter of $u$.

\section{PROBLEM FORMULATION}

Consider the $z$-directed uniform linear array lying on the $x$-axis with $K$ number of identical dipoles, with uniform spacing, an identical orientation and elements are electromagnetically and mutually coupled.

Assuming that the coupling of each dipole is affected by the $K-1$ dipoles, where $K$ is the number of dipoles, the mutual coupling matrix, $\mathbf{C}$, which is non-Toeplitz and non-banded, is of the form

$$
\mathbf{C}=\left[\begin{array}{cccc}
c_{1,1} & c_{1,2} & \ldots & c_{1, K} \\
c_{2,1} & c_{2,2} & \ldots & c_{2, K} \\
\vdots & \vdots & \ddots & \vdots \\
c_{K, 1} & c_{K, 2} & \ldots & c_{K, K}
\end{array}\right]
$$

The mutual coupling matrix in (5) is obtained by a "method of moments" (MoM) approximation (also known as the "boundary element method" (BEM) approximation of the solution of the Maxwell equations. This "method of moments" approximation software used is the "EMCoS Antenna VLab".

Under electromagnetic coupling, the linear array of antennas would give an array manifold of $\mathbf{a}^{(C)}(\theta):=\mathbf{C a}(\theta)$. This "coupled" array manifold would give $\mathbf{a}_{1}^{(C)}(u)$ and $\mathbf{a}_{2}^{(C)}(u)$, as counterparts to $\mathbf{a}_{1}(u)$ and $\mathbf{a}_{2}(u)$ of (3))-(4). In general, no scalar $\psi^{(C)}$ exists, such that $\psi^{(C)} \mathbf{a}_{1}^{(C)}(u)=\mathbf{a}_{2}^{(C)}(u)$. 
Under an idealization of $\mathbf{C}$ as Toeplitz and banded, C would equal

$$
\begin{aligned}
& \mathbf{C}^{(T B)} \\
& =\left[\begin{array}{lllllll}
c_{0} & c_{1} & \ldots & c_{Q} & 0 & \ldots & 0 \\
c_{-1} & c_{0} & \ldots & c_{Q-1} & c_{Q} & \ldots & 0 \\
\vdots & \vdots & \ddots & \vdots & \vdots & \ddots & \vdots \\
c_{-Q} & c_{-Q+1} & \ldots & c_{0} & c_{1} & \ldots & 0 \\
0 & c_{-Q} & \ldots & c_{-1} & c_{0} & \ldots & c_{Q} \\
\vdots & \vdots & \ddots & \vdots & \ddots & \vdots & \vdots \\
0 & \ldots & 0 & c_{-Q} & \ldots & c_{-1} & c_{0}
\end{array}\right]
\end{aligned}
$$

giving $\mathbf{a}_{1}^{(T B)}(u)$ and $\mathbf{a}_{2}^{(T B)}(u)$, as counterparts to $\mathbf{a}_{1}(u)$ and $\mathbf{a}_{2}(u)$ of (3)-(4). Even for this Toeplitz and banded special case of $\mathbf{C}$, no scalar $\psi^{(T B)}$ would generally exists such that $\psi^{(T B)} \mathbf{a}_{1}^{(T B)}(u)=\mathbf{a}_{2}^{(T B)}(u)$.

To restore the "rotational invariance" mathematical structure in $\mathbf{a}^{(C)}(\theta)$, [17] suggests discarding the data collected by the array's $Q$ number of leading antennas and $Q$ number of trailing antennas. These $2 Q$ are called "auxiliary" or "dummy" antennas.

To understand why this scheme would be effective: Suppose $Q$ number of "coupled" antennas, at either end of the linear array grid, have their data ignored. The remaining $(K-2 Q)$ "coupled" antennas may be formed into two $(K-2 Q-1)$-antenna subarrays. These two $(K-2 Q-1)$-antenna subarrays may be selected out from the overall $K$-element array by the two $(K-2 Q-1) \times K$ selection matrices,

$$
\begin{aligned}
& \mathbf{J}_{1} \\
& =\left[\mathbf{0}_{(K-2 Q-1) \times Q}, \mathbf{I}_{(K-2 Q-1)}, \mathbf{0}_{(K-2 Q-1) \times(Q+1)}\right], \\
& \mathbf{J}_{2} \\
& =\left[\mathbf{0}_{(K-2 Q-1) \times(Q+1)}, \mathbf{I}_{(K-2 Q-1)}, \mathbf{0}_{(K-2 Q-1) \times Q}\right] .
\end{aligned}
$$

These two $(K-2 Q-1)$-element subarrays of "coupled" antennas have the subarray manifolds,

$$
\begin{aligned}
& \mathbf{a}_{1}^{(Q)}(u) \\
= & \mathbf{J}_{1} \mathbf{C}^{(T B)} \mathbf{a}(u) \\
= & {\left[\begin{array}{c}
c_{-Q} \psi^{0}+c_{-Q+1} \psi^{1}+\ldots+c_{Q} \psi^{2 Q} \\
c_{-Q} \psi^{1}+c_{-Q+1} \psi^{2}+\ldots+c_{Q} \psi^{2 Q+1} \\
c_{-Q} \psi^{2}+c_{-Q+1} \psi^{3}+\ldots+c_{Q} \psi^{2 Q+2} \\
\vdots
\end{array}\right] } \\
:= & \mathbf{J}_{2} \mathbf{C}^{(T)}(u) \mathbf{a}(u) \\
= & {\left[\begin{array}{c}
c_{-Q} \psi^{1}+c_{-Q+1} \psi^{2}+\ldots+c_{Q} \psi^{2 Q+1} \\
c_{-Q} \psi^{2}+c_{-Q+1} \psi^{3}+\ldots+c_{Q} \psi^{2 Q+2} \\
c_{-Q} \psi^{3}+c_{-Q+1} \psi^{4}+\ldots+c_{Q} \psi^{2 Q+3} \\
\vdots
\end{array}\right] }
\end{aligned}
$$

where $\psi=\exp \left(j 2 \pi \frac{\Delta}{\lambda} u\right)$ as before, and

$$
\begin{aligned}
& \mathbf{J}_{1} \mathbf{C}^{(T B)}=\left[\begin{array}{llllll}
c_{-Q} & \ldots & c_{Q} & 0 & \ldots & 0 \\
0 & c_{-Q} & \ldots & c_{Q} & \ldots & 0 \\
\vdots & \vdots & \vdots & \vdots & \vdots & \vdots \\
0 & \ldots & c_{-Q} & \ldots & c_{Q} & 0
\end{array}\right], \\
& \mathbf{J}_{2} \mathbf{C}^{(T B)}=\left[\begin{array}{llllll}
0 & c_{-Q} & \ldots & c_{Q} & \ldots & 0 \\
\vdots & \vdots & \vdots & \vdots & \vdots & \vdots \\
0 & \ldots & c_{-Q} & \ldots & c_{Q} & 0 \\
0 & \ldots & 0 & c_{-Q} & \ldots & c_{Q}
\end{array}\right]
\end{aligned}
$$

It is obvious that $\mathbf{a}_{2}^{(Q)}(u)=\psi \mathbf{a}_{1}^{(Q)}(u)$ regardless of the value of $u$. In other words, a rotational invariance $(\psi)$ is restored despite Toeplitz/banded electromagnetic coupling among the antennas, but it is between the two $(K-2 Q-1)$-element subarrays obtained from the overall $K$-element array by ignoring $Q$ number of "auxiliary"/"dummy" antennas at either end of the linear array grid.

The above formulation will be applied to varying dipoles' electrical lengths in order to know its effects on the direction-of-arrival estimation under mutual coupling.

\section{SIMULATION}

In order to obtain the numerical values of (5), the uniform linear antenna model is set up and run in VLab. Throughout the simulation, dipoles have a diameter of $0.6 \lambda$ millimeters, a feeding gap of $\frac{\lambda}{50}$. They are center-driven by a one-volt source with a load impedance of $z_{o}=50 \mathrm{ohms}$, and mechanically in free-space. $\lambda$ refers to the wavelength.

For the test case of a linear array of $K=11$ identical dipoles spaced uniformly apart by $\frac{\Delta}{\lambda}=0.5$, the above VLab computation produces $K \times K$ mutual coupling matrix $\mathbf{C}$ for varying electrical dipole lengths: $\frac{L}{\lambda}=0.30,0.50,0.65,0.80,0.95$.

Fig. 1 and Fig. 2 show the three-dimensional plots of the real and imaginary parts of $\mathbf{C}$ from VLab at $\frac{L}{\lambda}=0.65$ and $\frac{L}{\lambda}=0.80$, respectively. If written in matrix form, Cs are symmetric matrices and not Toeplitz and banded, for all $\frac{L}{\lambda}$. As the symmetry is along the diagonal, the impedance of the diagonal elements are called the "self-impedance", while the impedance of the elements off the diagonal are called the "mutual impedance".

For direction-of-arrival estimation, there is only one incident source, incident from the far field, emitting a narrowband signal whose baseband-equivalent amplitude envelope is modeled as symmetrically complex-value Gaussian and temporally uncorrelated. The signal-to-noise ratio (SNR) is $20 \mathrm{~dB}$. Each icon in Fig. 3 represents 100 independent Monte Carlo trials, each of which involves 500 time samples. 


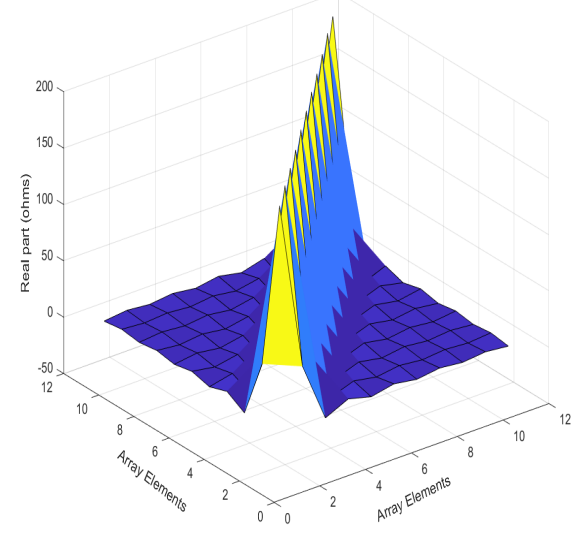

(a)

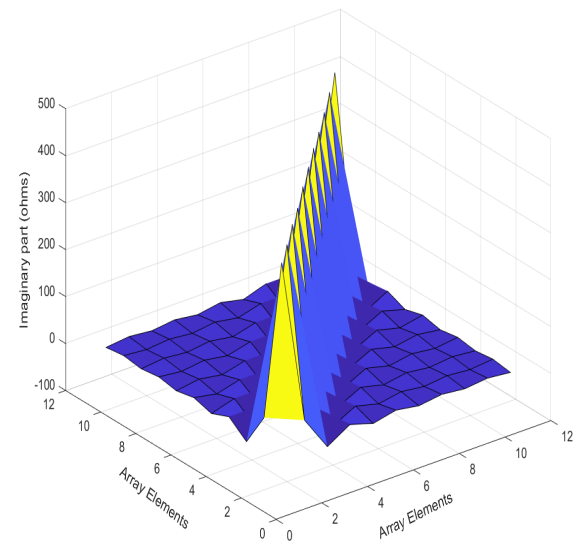

(b)

Figure 1: (a) Real and (b) imaginary parts of $\mathbf{C}$ at $\frac{L}{\lambda}=$ 0.65

The direction-of-arrival estimation performance is measured by the root mean square error (RMSE) in (7).

$$
R M S E=\sqrt{\frac{\sum_{k=1}^{K}\left(\theta_{k}-\hat{\theta}_{k}\right)^{2}}{K}}
$$

where $K$ denotes the number of elements in the uniform linear array, while $\theta$ and $\hat{\theta}$ denote the true direction of arrival and the estimate, respectively.

\section{RESULTS AND DISCUSSION}

Using the ESPRIT-based algorithm in [17], Fig. 3 shows the results of the direction-of-arrival estimation. The "root mean square error" per $\frac{L}{\lambda}$ is plotted against the "number of auxiliary sensors".

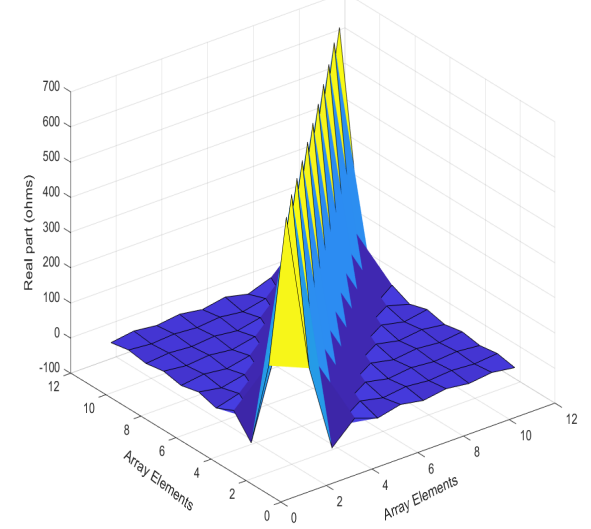

(a)

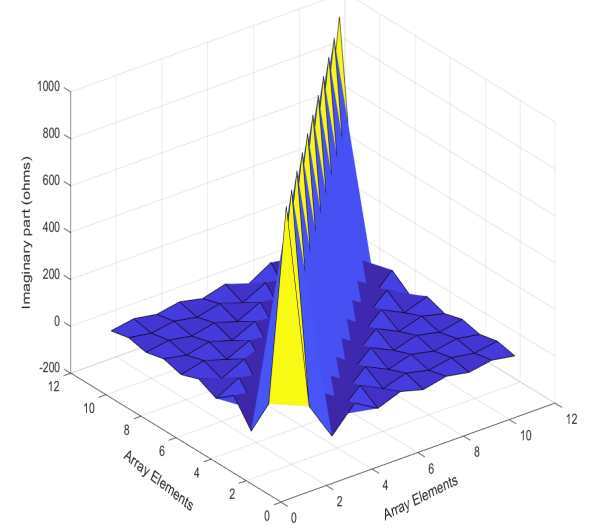

(b)

Figure 2: (a) Real and (b) imaginary parts of $\mathbf{C}$ at $\frac{L}{\lambda}=$ 0.80

Two cases are considered here. The first case is when $\mathbf{C}=\mathbf{C}^{(T B)}$, i.e. $\mathbf{C}$ is presumed as Toeplitz and banded. The second case is when $\mathbf{C} \neq \mathbf{C}^{(T B)}$, i.e. $\mathbf{C}$ is VLab's coupling matix. The first case is reflected on the curves with solid lines in Fig. 3. They are based on a $\mathbf{C}^{(T B)}$ obtained by least-squares fitting VLab's $\mathbf{C}$ values to the $\mathbf{C}^{(T B)}$ mathematical form. The second case is presented by the curves with dashed lines in Fig. 3. 


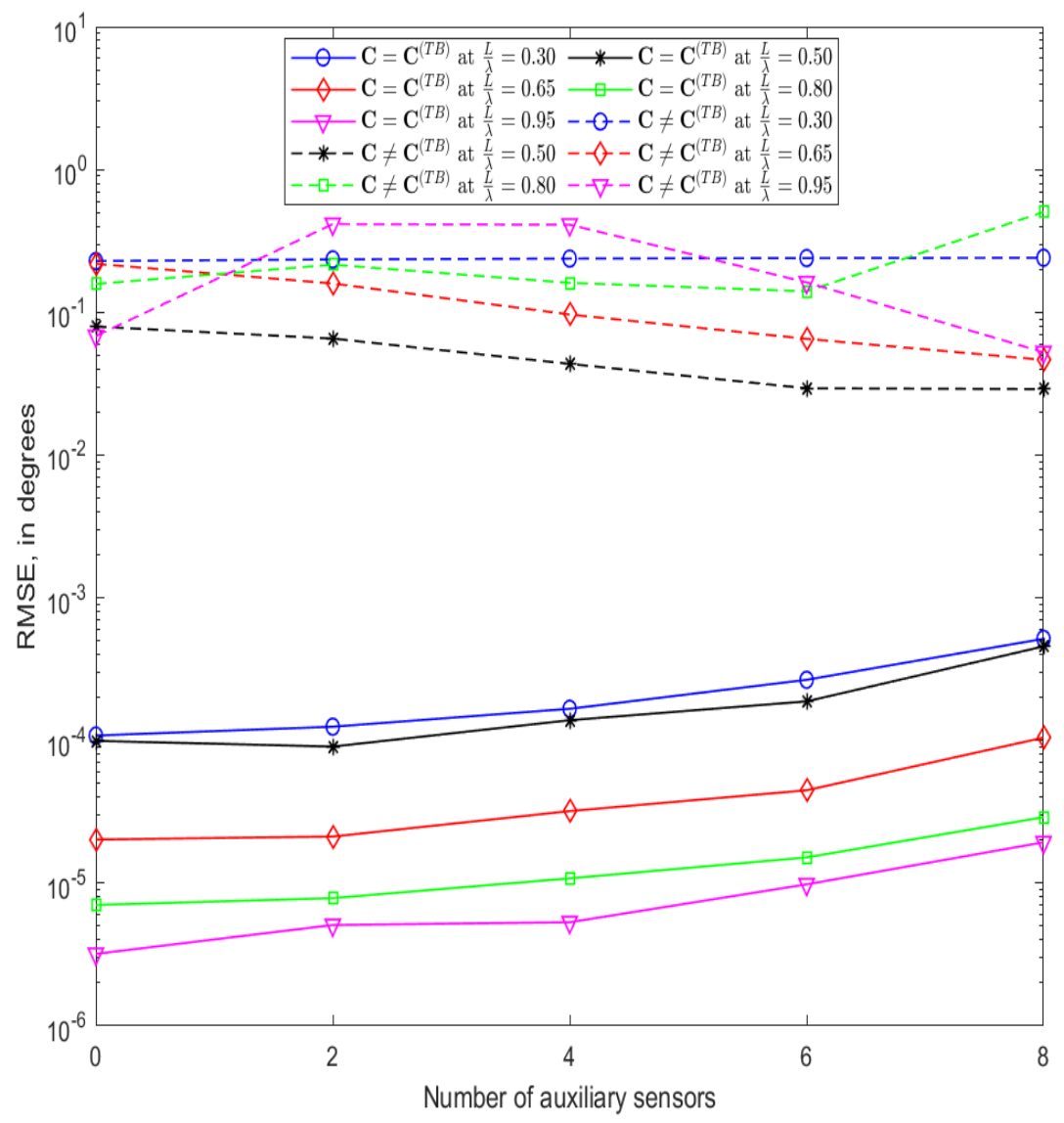

Figure 3: The root mean square error versus the number of auxiliary sensors at $\frac{L}{\lambda}=0.30,050,0.65,0.80,0.95$.

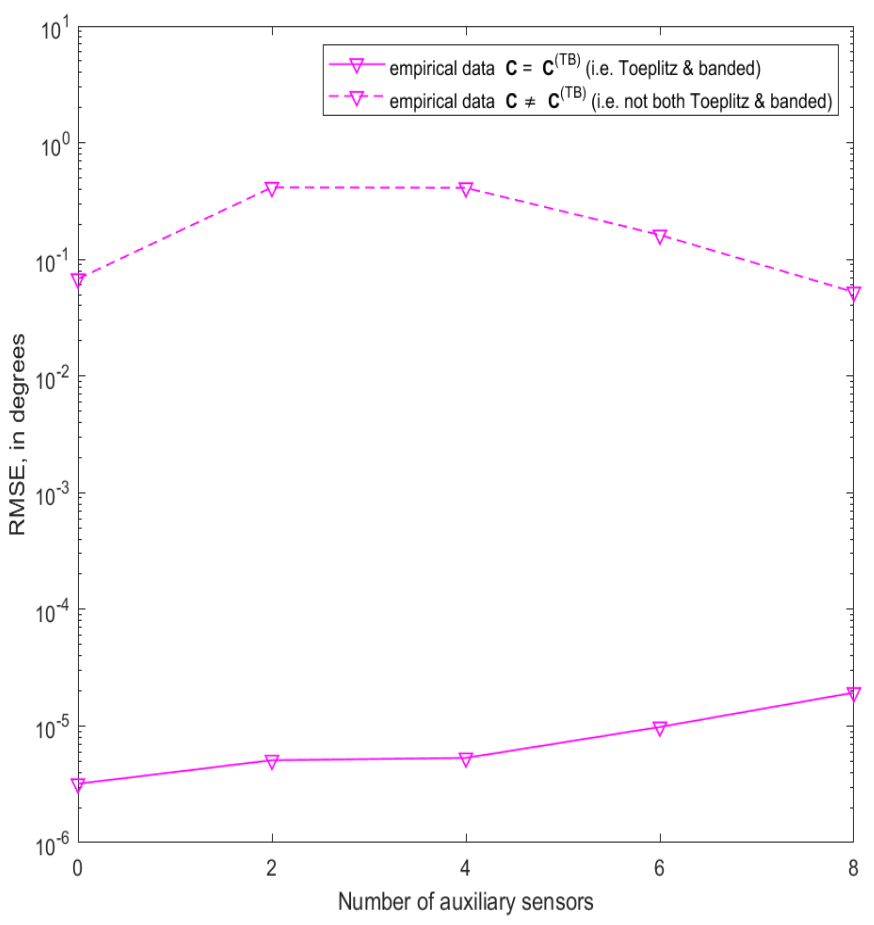

Figure 4: The root mean square error versus the number of auxiliary sensors at $\frac{L}{\lambda}=0.95$. 
For the case when $\mathbf{C}=\mathbf{C}^{(T B)}$, good performance in direction-of-arrival estimations is expected since the Toeplitz and banded the mutual coupling matrix is presumed. For all $\frac{L}{\lambda}$, the root mean square error improves when fewer "auxiliary" sensors considered decreases. Fewer auxiliary antennas results to a larger mid-array aperture. This is valid because in sensor array direction finding, more accurate direction-ofarrival (DOA) estimates and more robust resolution of closely spaced emitters would be produced if the antenna array aperture is larger.

Increasing the $\frac{L}{\lambda}$ improves the root mean square error, thus improving the performance of direction-ofarrival estimation. The performance improved from an order of magnitude of -4 to -6 at $Q=0$, and from an order of magnitude of -4 to -5 at $Q=4$ across all $\frac{L}{\lambda}$. This observation is correct, since longer electrical dipole lengths would warrant a larger "effective area" of the antenna array, thereby capturing more power from a given electromagnetic field.

When the algorithm in [17] is fed with a more realistic data, i.e. $\mathbf{C} \neq \mathbf{C}^{(T B)}$, the direction-of-arrival estimation performance is very poor across all $\frac{L}{\lambda}$ in Fig. 3. Fig. 4 shows the worst performance where the two curves differ by 3 to 5 orders of magnitude. The increase in $\frac{L}{\lambda}$, in this case, would not merit further analysis since the presumed model of the mutual coupling matrix being Toeplitz and banded is not followed, in the first place.

\section{CONCLUSION}

Varying the electrical dipole lengths in the uniform linear array improves the performance of the direction-of-arrival estimation as longer dipoles collectively provides larger aperture or receiving cross section of the antenna array. This translates to more power being captured by the array. As far as discarding the mutual coupling data from both ends of the uniform linear array, fewer dipoles considered as "auxiliary" antennas would translate to a larger midarray aperture, which in turn produce more accurate direction-of-arrival estimates.

This paper also presented the serious consequence of mis-modeling the mutual coupling matrix as both Toeplitz and banded. Feeding the ESPRIT-based algorithm in [17] with a more practical data results to an inaccurate direction-of-arrival estimation. The next step is to develop a mathematically tractable coupling model that is valid and can be used in the effective mitigation of electromagnetic mutual coupling among the antenna array elements.

\section{References:}

[1] R. Roy \& T. Kailath, "ESPRIT-Estimation of Signal Parameters Via Rotational Invariance
Techniques," IEEE Transactions on Acoustics, Speech, and Signal Processing, Vol. 37, No. 7, July 1989, pp. 984-995.

[2] S. Lundgren, "A study of mutual coupling effects on the direction finding performance of ESPRIT with a linear microstrip patch array using the method of moments," Antennas and Propagation Society International Symposium, Vol. 2, 1996, pp. 1372-1375.

[3] F. I. Tseng \& J. Venkataraman, "Optimization for adaptive antenna arrays with mutually coupled elements via spatially smoothed ESPRIT," IEEE Topical Conference on Wireless Communication Technology, 2003, pp. 376-377.

[4] Y. Inoue \& H. Arai, "Effect of mutual coupling and manufacturing error of array for DOA estimation of ESPRIT algorithm," Electronics and Communications in Japan, part 1, Vol. 89, 2006, No. 9, pp. 68-76.

[5] B. Himed \& D. D. Weiner, "A generalized treatment of mutual coupling compensation for ESPRIT," IEEE International Symposium on Circuits and Systems, Vol. 1, 1989, pp. 296-299.

[6] B. Himed \& D. D. Weiner, "Compensation of mutual coupling effects for the ESPRIT algorithm," IEEE Antennas and Propagation Society International Symposium, Vol. 2, 1989, pp. 966-969.

[7] B. Himed \& D. D. Weiner, "Compensation for mutual coupling effects in direction finding," IEEE International Conference on Acoustics, Speech, and Signal Processing, Vol. 5, 1990, pp. 2631-2634.

[8] T. T. Zhang \& H. T. Hui, "Direction of arrival estimation using ESPRIT with mutual coupling compensation," IEE Proceedings: Science, Measurement and Technology, Vol. 151, No. 6, November 2004, pp. 476-478.

[9] T. T. Zhang, H. T. Hui \& Y. L. Lu, "Compensation for the mutual coupling effect in the ESPRIT direction finding algorithm by using a more effective method," IEEE Transactions on Antennas and Propagation, Vol. 53, No. 4, April 2005, pp. 1552-1555.

[10] S. Hwang, S. Burintramart, T. K. Sarkar \& S. R. Best, "Direction of arrival (DOA) estimation using electrically small tuned dipole antennas," IEEE Transactions on Antennas and Propagation, Vol. 54, No. 11, November 2006, pp. 32923301. 
[11] L. Xiaohua, Z. Jianjiang, L. Hailin \& C. Wenqi, "Compensation for the mutual coupling effect for the ESPRIT algorithm in single-snapshot array processing," Journal of Electronics (China), Vol. 24, No. 5, September 2007, pp. 662-667.

[12] J. E. F. del Rio, O. M. Conde-Portilla \& M. F. Catedra, "Estimating azimuth and elevation angles when mutual coupling is significant," $A n$ tennas and Propagation Society International Symposium, Vol. 1, 1998, pp. 215-218.

[13] T. Svantesson, "Modeling and estimation of mutual coupling in a uniform linear array of dipoles," IEEE International Conference on Acoustics, Speech, and Signal Processing, Vol. 5, 1999, pp. 2961-2964.

[14] X. Guo, Q. Wan \& W. Yang, "Parameters estimation of coherently distributed sources in the presence of mutual coupling," International Conference on Signal Processing, Vol. 1, 2006.

[15] Z. Ye \& C. Liu, "2-D DOA estimation in the presence of mutual coupling," IEEE Transactions on Antennas and Propagation, Vol. 56, No. 10, October 2008, pp. 3150-3158.

[16] A. Azarbar, G. R. Dadashzadeh \& H. R. Bakhshi, "Non-sensitive matrix pencil method against mutual coupling," IEICE Electronics Express, Vol. 8, No. 6, March 2011, pp. 318-324.

[17] H.-b. Li, Y.-d. Guo, J. Gong \& J. Jiang, "Mutual coupling self-calibration algorithm for uniform linear array based on ESPRIT," International Conference on Consumer Electronics, Communications and Networks, 2012, pp. 3323-3326.

[18] J.-G. Hong, W.-H. Ahn \& B.-S. Seo, "Compensation of mutual coupling in an antenna array for direction of arrival estimation," International Conference on Advanced Communication Technology, 2013, pp. 599-603.
[19] W. Si, D. Wu, L. Liu \& X. Qu, "Direction finding with gain/phase errors and mutual coupling errors in the presence of auxiliary sensors," Mathematical Problems in Engineering, 2014, pp. 1-12.

[20] Y. I. Wu, G. P. Arada, W. Tam \& K. T. Wong, "Mis-modeling and mis-correction of mutual coupling in an antenna array - A case study in the context of direction finding using a linear array of identical dipoles," IEEE International Conference on Signal and Image Processing, 2016, pp. 447-451.

[21] Y. I. Wu, G. P. Arada, K. T. Wong \& W.-Y. Tam, "Electromagnetic coupling matrix modeling and ESPRIT-based direction finding - A case study using a uniform linear array of identical dipoles," IET International Conference on Intelligent Signal Processing, 2015, pp. 1-5.

[22] K.T. Wong, Y. Song, C. J. Fulton, S. Khan \& W.-Y. Tam, "Electrically "long" dipoles in a collocated/orthogonal triad - for direction finding and polarization estimation," IEEE Transactions on Antennas and Propagation, Vol. 65, No. 11, November 2017, pp. 6057-6067.

[23] S. Khan \& K. T. Wong, "Electrically long dipoles in a crossed pair for closed-form estimation of an incident source's polarization," IEEE Transactions on Antennas and Propagation, Vol. 67, No. 8, August 2019, pp. 55695581 .

[24] S. Khan \& K. T. Wong, "A six-component vector sensor comprising electrically long dipoles and large loops - to simultaneously estimate incident sources' directions-of-arrival and polarizations," IEEE Transactions on Antennas and Propagation, Vol. 68, No.8, August 2020, pp. 6355-6363.

\section{Creative Commons Attribution License 4.0 (Attribution 4.0 International, CC BY 4.0)}

This article is published under the terms of the Creative Commons Attribution License 4.0 https://creativecommons.org/licenses/by/4.0/deed.en_US 